\title{
CRITICAL ISCHEMIA OF THE FINGERS IN AN AUTO MECHANIC AS A RESULT OF OCCUPATIONAL EXPOSURE
}

\author{
MACIEJ RABCZYŃSKI ${ }^{1}$, EDWIN KUŹNIK ${ }^{1}$, MACIEJ GUZIŃSKI ${ }^{2}$, and RAJMUND ADAMIEC ${ }^{1}$ \\ ${ }^{1}$ Medical University in Wroclaw, Wrockaw, Poland \\ Department of Angiology, Hypertension and Diabetology \\ ${ }^{2}$ Medical University in Wroclaw, Wrocław, Poland \\ Department of Radiology, Institute of General Radiology, Interventional Radiology and Neuroradiology
}

\begin{abstract}
Hypothenar hammer syndrome is a rare cause of ischemic fingers observed mainly in young men smoking cigarettes and it is associated with repeated trauma of the ulnar artery in the area of the hypothenar eminence of the dominant-hand arm, resulting in a deficit of blood supply with the occurrence of hand symptoms typical for chronic and sometimes critical ischemia. Artery injury in this location is most often the result of multiple repetitions of the same activity being mostly the result of occupational exposure. We present a case of a 27-year-old car mechanic admitted to the hospital with symptoms of critical ischemia of the fingers III, IV, and V of the right hand, which resolved after conservative treatment.
\end{abstract}

Key words:

Occupational exposure, HHS, Critical ischemia, Vascular disease

\section{INTRODUCTION}

Twenty-seven-year-old man who worked as a car mechanic was admitted to the Department of Angiology, Arterial Hypertension and Diabetology of the Wroclaw Medical University due to critical ischemia of the fingers of the dominant right arm. The main complaints were related to resting pain in finger III, IV and V, accompanied by bruising and cold skin. During the interview, the patient did not report any significant past diseases. Approximately 7 weeks prior to the admittance to the hospital, there was a sudden bruising of the right hand fingertips associated with pain of moderate intensity, numbness, paresthesia, and decreased skin temperature. In connection with the periodic escalation of the symptoms, the patient was consulted 3 times by the acute vascular on-call team, where he received a recommendation for the empirical use of low molecular weight heparin. He was admitted to the hospital with the initial diagnosis of Buerger's disease, as confirmed by young age and a history of tobacco addiction, due to the persistence of the symptoms.

On admission, the patient was in good general condition, without any circulatory or respiratory impairment. Subjective clinical findings included resting pain of the fingers III, IV and V of the right hand requiring treatment with the use of opioid analgesics. Clinical inspection showed bruising, coolness of the skin of the fingers

Received: October 29, 2013. Accepted: May 16, 2014.

Corresponding author: M. Rabczyński, Medical University in Wroclaw Department of Angiology, Hypertension and Diabetology, Borowska 213, 50-333 Wrocław, Poland (e-mail: maciejrabczynski@gmail.com). 
III, IV and V of the right hand, callus of the epidermal tip of the previously mentioned fingers, and the presence of trophic changes on the tip of the III finger. The Allen test was positive at the distal end of the right radial artery. Pulse in the upper extremities, including the ulnar artery and the radial artery, and in locations typical for the lower extremities was palpable; there was no asymmetry in blood pressure.

The results of laboratory tests showed only mediocre hypercholesterolemia (total cholesterol $=261 \mathrm{mg} / \mathrm{dl}$, norm: 130-200 mg/dl). The diagnosis of antiphospholipid syndrome (anti-cardiolipin and anti- $\beta 2$-glycoprotein antibodies, lupus anticoagulant), systemic vasculitis (pANCA, cANCA, cryoglobulin), and systemic connective tissue disease (RF, ANA) were negative. Moderate hyperhomocysteinemia was then re-confirmed (19.24 $\mu \mathrm{mol} / \mathrm{l}$, norm: 5.46-16.2 $\mu \mathrm{mol} / \mathrm{l})$.

An investigation of the cardiovascular system for peripheral embolism was performed. Transthoracic echocardiography revealed no thrombus; an examination with the use of air contrast excluded the possibility of heart defect with a shunt. An X-ray examination ruled out abnormalities of the cervicothoracic region and bone structures of the right hand. No flow spectrum disorders of the arteries and veins of the upper limbs on both sides were found on Duplex Doppler test. No abnormalities were detected either in the vessels supplying the brain. A segmental Doppler pressure measurement on the arteries of the upper limbs showed no pathology. The findings of capillaroscopy were nonspecific; the observed changes suggested ischemia.

The detailed assessment of the peripheral vascular system of the right upper limb, especially the hand, was further augmented by 64-slice CT angiography (Photo 1 and 2). It revealed normal forearm arteries without atherosclerotic lesions and stenosis of the lumen; no aneurysms of the arm and forearm artery were present, either. However, tortuosity of the distal ulnar artery within the hypothenar eminence, after leaving the deep palmar branch and

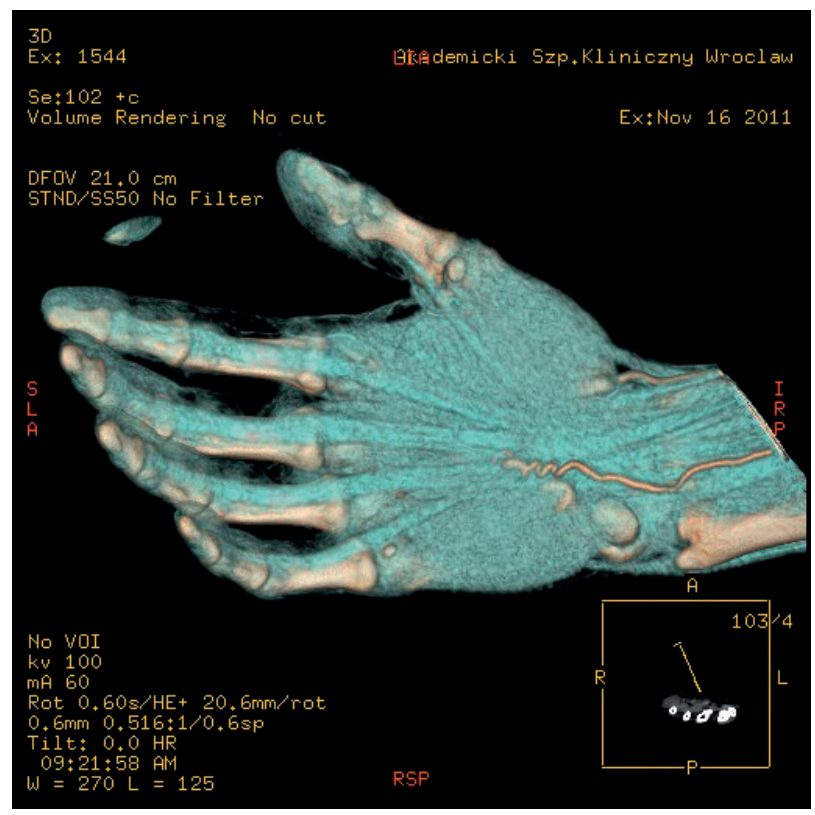

Photo 1. Right-hand computed tomography angiography (CTA) volume rendering reconstruction, corkscrewing course of the ulnar artery to the length of about $3 \mathrm{~cm}$

before going to superficial palmar arch, was visualized. In this area, the ulnar artery demonstrated a slight expansion of the lumen to about $2.5 \mathrm{~mm}$ with an accompanying serpentine "corkscrewing" course and angular folds to the length of about $3 \mathrm{~cm}$. Metacarpophalangeal arterioles to finger III, IV and V were rated as narrow, but unobstructed. Based on the data from the anamnesis, reported symptoms, physical examination, and additional results, the diagnosis of hypothenar hammer syndrome was established. The consulting vascular surgeon did not see indications for surgical treatment. Conservative treatment, including low molecular weight heparin, acetylsalicylic acid, and intravenous infusions of bencyclan, was proposed. Gradual withdrawal of the symptoms and pathological changes was obtained. Furthermore, cessation of smoking and avoidance of further mechanical injuries to this area of the hand were also recommended.

The patient remains in ambulatory follow-up. The observed symptoms gradually resolved after 2 months, there were no signs of a blood flow deficit in terms of fingers. 


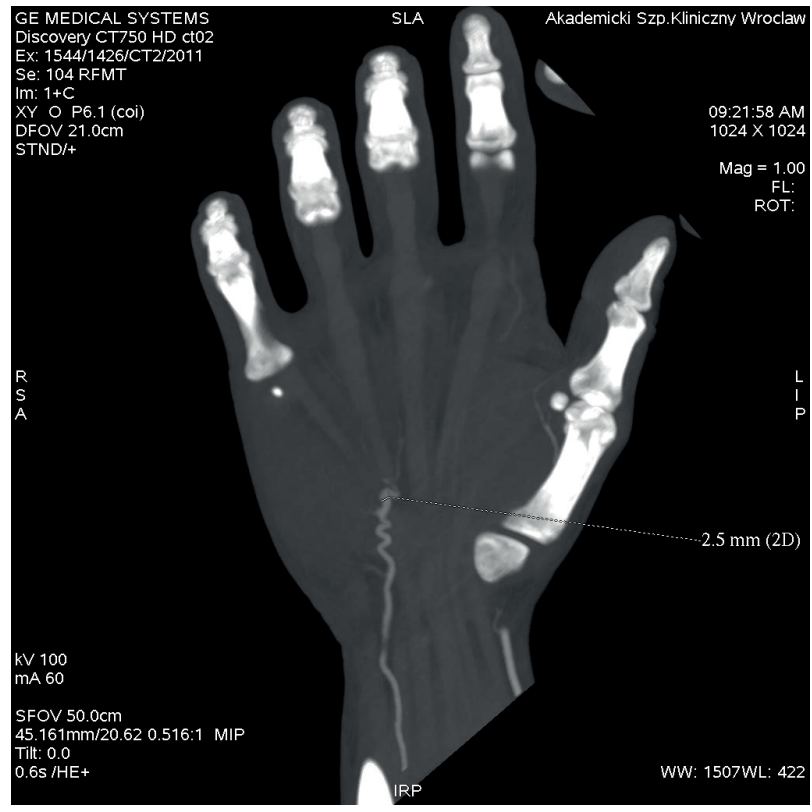

Photo 2. Right-hand computed tomography angiography (CTA) maximum intensity projection (MIP reconstruction), corkscrewing course of the ulnar artery to the length of about $3 \mathrm{~cm}$

\section{DISCUSSION AND CONCLUSIONS}

Hypothenar hammer syndrome (HHS) is a rare cause of ischemic fingers. HHS was first defined relatively recently (1970, Conn et al.) [1]. HHS usually occurs in the dominant hand of young male smokers. Chronic traumas to the hypothenar eminence in professionally predisposed individuals (drivers, mechanics, bricklayers, carpenters), or those engaged in a number of sports (such as golf, tennis, extreme martial arts, baseball in Anglo-Saxon countries) cause chronic pressure of the ulnar artery against the hamulus (uncinate process) of the hamate bone [2-4]. The ulnar artery, and sometimes the ulnar nerve too, is compressed between the muscles of the hypothenar eminence, which act as the 'hammer', and the hamate bone, which acts as the 'anvil', resulting in the ischemia of the fingers via 2 mechanisms.

Firstly, ulnar artery aneurysm, which may be a source of peripheral arterio-arterial emboli can occur. Secondly, direct arterial endothelium damage may lead to arterial thrombosis. The ischemic symptoms described in the literature vary in severity from chronic, manifesting when the hand is challenged by an increased effort, to critical with the presence of resting pain or trophic finger changes, which may lead to amputation of the fingers [2,5,6]. Neurological symptoms (pain, paresthesia, numbness) occur as a result of concomitant ulnar nerve injury. In the hand, the ulnar nerve runs segmentally together with the ulnar artery in the ulnar eminence created by the hamulus of the hamate bone, pisiform bone, and transverse carpal ligament, which form the anatomical Guyon's canal [7-9].

The natural course of the disease is usually similar. There are no prodromal symptoms because of rich palmar vasculature. All described signs occur in case of thrombotic occlusion or are connected with thrombotic embolization. Administration of an anticoagulant treatment may reduce the risk of finger gangrene development.

Only descriptions of individual cases of this syndrome are present in literature due to its low incidence (the second case in our department) [10]. Moreover, studies conducted in the population at risk of exposure allow for diagnosing HHS with medium severity of symptoms. Kaji et al. [11] indicate the presence of HHS in $9 \%$ of 330 employees whose work was associated with exposure to vibration. Little and Ferguson [12] demonstrated clinical and Doppler signs of HHS in 11 of 79 examined auto mechanics (14\%). The differential diagnosis should include other, more common, causes of ischemic hands: Buerger's disease, primary and secondary Raynaud's phenomenon, systemic vasculitis, cardiac embolism, pulmonary arterio-arterial, arterio-arterial embolism, congenital or acquired hypercoagulability (thrombophilia), and thoracic outlet syndrome. In the presented case, young age and smoking suggested a diagnosis of Buerger's disease, but the presence of the pulse peripherally on the upper and lower limbs and normal forearm arteries visualized in ultrasound and CT angiography argued against this diagnosis. "Corkscrew" appearance of the ulnar artery in the palmar segment 
is characteristic for HHS. The young age of the patient virtually ruled out atherosclerosis and its complications as the cause of the observed clinical picture.

In the thoracic outlet syndrome (TOS), neurological symptoms dominate over the ischemic ones. Also, TOS affects women twice as often as men, typically in the fourth decade of life, and the symptoms usually appear in connection with the adoption of a specific position of the upper limbs (lifting, adduction). In the course of clinically significant occlusion of the artery, asymmetric pulse of the radial and ulnar arteries with the difference in the measurement of blood pressure greater than $20 \mathrm{~mm} \mathrm{Hg}$ are usually observed. In the present case, there was no evidence that the severity of symptoms depends on the position of the limbs, nor was there any asymmetry in the blood pressure of the upper extremities.

Atypical image in capillaroscopy and nonparoxysmal nature of the observed changes in the fingers without the characteristic phasic occurrence did not allow for recognizing primary Raynaud's phenomenon. Secondary causes were excluded by laboratory tests (no activation of the immune system, negative autoantibodies). Similarly, antiphospholipid syndrome was also excluded. The authors have observed, however, cases of iatrogenic closure of the radial and ulnar artery thanks to the previously performed angiography/angioplasty via the upper limb access, leading to arterial thrombosis as a result of endothelial activation. The unquestionable progress in radiological diagnostics provides very accurate assessment of the vascular anatomy. Angiography remains the gold standard; nevertheless, high resolution computed tomography, being a less invasive test, seems to have a growing importance. In our case, it was limited to the implementation of the 64-row CT angiography with a resolution of $0.6 \mathrm{~mm}$. In the authors' opinion, angiography should be performed in the absence of conclusive results of computed tomography, or in the case where endovascular surgical procedure (distal embolization with the formation of trophic changes) is a treatment option.
Hypothenar hammer syndrome treatment should be adapted to the clinical picture and the severity of the local status of the fingers. Conservative treatment is mentioned in the majority of HHS cases in literature. The mainstay of treatment is to prevent further mechanical damage. In the vast majority of patients, long-term smoking or active smoking is noted in the history. The association between nicotine addiction and the pathogenesis of HHS is still unclear; however, smoking cessation is a necessity. In the absence of necrosis, a conservative therapy using low molecular weight heparin, antiplatelet agents, thrombolytic agents, and vasoactive agents (prostaglandin E1, sulodexide, pentoxifylline, bencyclane) is sufficient $[7,13,14]$.

Surgical approach consists of thrombectomy or, if necessary, reconstructive treatment (arterial autograft, autologous venous bypass) [15,16], and is reserved for cases of critical ischemia with the presence of severe pain and advanced regressive changes in the affected fingers. Cases of HHS treated using the da Vinci robot [17] or by selective sympathetic denervation with botulinum toxin A [7] have been described, as well.

Hypothenar hammer syndrome is a rare vascular disorder. Usually, young workers are affected and the disease is connected with their professional work. The lack of prodromal symptoms did not allow for indicating the persons at risk. That is why the use of prevention measures (e.g., protective gloves) is suggested in professionally predisposed individuals who are at risk of repeated trauma to the hypothenar eminence.

\section{REFERENCES}

1. Conn J, Bergan JJ, Bell JL. Hypothenar hammer syndrome: Posttraumatic digital ischemia. Surgery. 1970;68:1122-8.

2. Jagenburg A, Goyen M, Hirschelmann R, Carstens IM, Kröger K. Hypothenar hammer syndrome: Causes, sequelae and diagnostic aspects. Fortschr Röntgenstr. 2000;172: 295-300, http://dx.doi.org/10.1055/s-2000-115. 
3. Mueller LP, Mueller LA, Degreif J, Rommens PM. Hypothenar hammer syndrome in a golf player. A case report. Am J Sports Med. 2000;28:741-5, http://dx.doi. org/10.1177/28.5.741-a.

4. Noel B, Hayoz D. A tennis player with hand claudication. Vasa. 2000;29:151-3, http://dx.doi.org/10.1024/03011526.29.2.151.

5. Yuen JC, Wright E, Johnson LA, Culp WC. Hypothenar hammer syndrome: An update with algorithms for diagnosis and treatment. Ann Plast Surg. 2011;67:429-38, http:// dx.doi.org/10.1097/SAP.0b013e31820859e1.

6. Keo HH, Umer M, Baumgartner I, Willenberg T, Gretener SB. Long-term clinical outcomes in patients diagnosed with severe digital ischemia. Swiss Med Wkly. 2011;141: w13159, http://dx.doi.org/10.4414/smw.2011.13159.

7. Swanson KE, Bartholomew JR, Paulson R. Hypothenar hammer syndrome: A case and brief review. Vasc Med. 2012;17:108-15, http://dx.doi.org/10.1177/ $1358863 X 11425878$.

8. Duncan WC. Hypothenar hammer syndrome: An uncommon cause of digital ischemia. J Am Acad Dermatol. 1996; 34:881-3, http://dx.doi.org/10.1016/S0190-9622(96)90070-5.

9. Vayssairat M, Debure C, Cormier JM, Bruneval P, Laurian C, Juillet Y. Hypothenar hammer syndrome: Seventeen cases with long-term follow-up. J Vasc Surg. 1987;5:838-43, http://dx.doi.org/10.1016/0741-5214(87)90096-6.

10. Kluz J, Małecki R, Adamiec R. Hypothenar hammer syndrome complicated by critical finger ischemia in a fight sports instruktor. Acta Angiol. 2006;12:180-6.
11. Kaji H, Honma H, Usui M, Yasuno Y, Saito K. Hypothenar hammer syndrome in workers occupationally exposed to vibrating tools. J Hand Surg Br. 1993;6:761-6, http:// dx.doi.org/10.1016/0266-7681(93)90240-G.

12. Little JM, Ferguson DA. The incidence of the hypothenar hammer syndrome. Arch Surg. 1972;105:684-5, http:// dx.doi.org/10.1001/archsurg.1972.04180110009004.

13. Wieczorek I, Farber A, Alexander K. Hypothenar hammer syndrome successfully managed with intravenous prostaglandin E1 and heparin and with correction of the thrombogenic risk profile. A case report. Angiology. 1996;47:1111-6, http://dx.doi.org/10.1177/000331979604701113.

14. Yakubov SJ, Nappi JF, Candela RJ. Successful prolonged local infusion of urokinase for the hypothenar hammer syndrome. Cathet Cardiovasc Diagn. 1993;29:301-3, http:// dx.doi.org/10.1002/ccd.1810290413.

15. Smith HE, Dirks M, Patterson RB. Hypothenar hammer syndrome: Distal ulnar artery reconstruction with autologous inferior epigastric artery. J Vasc Surg. 2004;40:1238-42, http://dx.doi.org/10.1016/j.jvs.2004.09.031.

16. Mousa AY, Stone PA, Nanjundappa A, Campbell JE, AbuRahma AF. Hypothenar hammer syndrome in a 22-year-old male patient: A case report and review of literature. Vascular. 2012;20:100-3, http://dx.doi.org/10.1258/ vasc.2011.cr0272.

17. Facca S, Liverneaux P. Robotic assisted microsurgery in hypothenar hammer syndrome: A case report. Comput Aided Surg. 2010;15:110-4, http://dx.doi.org/10.3109/10929088. 2010.507942 .

This work is available in Open Access model and licensed under a Creative Commons Attribution-NonCommercial 3.0 Poland License - http://creativecommons.org/ licenses/by-nc/3.0/pl/deed.en. 